\title{
Changes in intraocular pressure during low dose intravenous sedation with propofol before cataract
} surgery

\author{
Sean Neel, Robert Deitch Jr, S S Moorthy, Stephen Dierdorf, Robert Yee
}

\begin{abstract}
Aims-This study examined the effects on intraocular pressure, pulse rate, and blood pressure of low dose intravenous sedation with propofol.

Methods-Twenty adult patients who were scheduled to undergo cataract surgery were given a single intravenous bolus (0.98 (SEM 0.4$) \mathrm{mg} / \mathrm{kg}$ ) of propofol for sedation before administering the local anaesthetic for cataract surgery. A small intravenous dose of lignocaine was the only other anaesthetic or sedative agent given. The intraocular pressure in the non-surgery eye, the pulse rate, and the blood pressure were measured before and after propofol induction.

Results-Compared with the preinduction baseline, there was a $17 \%$ to $27 \%$ (from 16.2 (0.7) $\mathrm{mm} \mathrm{Hg}$ to $11.8(0.7) \mathrm{mm}$ $\mathrm{Hg})$ decrease in intraocular pressure after propofol induction. A significant decrease in intraocular pressure occurred within the first minute and was still evident at 7 minutes when the measurements were stopped. There was also a $7 \%-12 \%$ increase in pulse rate during the first 4 minutes, a $12 \%$ decrease in mean systolic blood pressure, and a 7\% decrease in mean diastolic blood pressure from baseline after propofol induction.

Conclusion-A single low dose bolus of propofol used for sedation before cataract surgery caused a moderate reduction in intraocular pressure with minimal, easily managed side effects.
\end{abstract}

(Br f Ophthalmol 1995; 79: 1093-1097)

Propofol is a non-barbiturate intravenously administered hypnotic/sedative agent that is commonly used in the USA for short term sedation before ophthalmic surgery and less commonly for examinations under anaesthesia. Propofol is an excellent choice for this because it produces rapid induction, can be used for maintenance anaesthesia if needed, and has a rapid, smooth recovery to a clear thinking state. ${ }^{12}$ In addition, sedation with propofol has a low incidence of nausea and vomiting, and the common side effects of propofol can be minimised and readily managed. ${ }^{1}$ Propofol's sedative effect is dose dependent with low doses causing sedation, drowsiness, and decreased responsiveness. ${ }^{1}$ At moderate doses hypnosis occurs and at high doses $(2 \cdot 0-2 \cdot 5 \mathrm{mg} / \mathrm{kg})$ anaesthesia (unconsciousness) occurs. ${ }^{1}$
Before ophthalmic surgery, intravenous sedation can be used to allow the ophthalmologist to give a retrobulbar and facial nerve block. Local anaesthetics have the advantage of having lower risks of morbidity and mortality, lower monetary costs, and less recovery time after day surgery compared with general anaesthesia and continuous intravenous anaesthesia. A disadvantage of local blocks is that it is painful to receive the injection. Propofol can be used to sedate the patient so that the local blocks may be given with minimal patient discomfort, minimal patient movement, and so that the patient does not remember receiving the block. Previous studies have determined that propofol is equal to, or superior to, other agents in providing adequate sedation and amnesia for retrobulbar and peribulbar blocks. ${ }^{3}$

Elevated intraocular pressure before intraocular surgery is associated with an increased risk of surgical complications. It is well known that a normal to slightly lower than normal intraocular pressure is desirable before and during surgery. To minimize these risks, the agent used for intravenous sedation should not raise the intraocular pressure. Since propofol can be administered before intraocular surgery, it is important to know the effect of low dose sedation with propofol on intraocular pressure.

Many previous studies have evaluated the effect on intraocular pressure of a variety of anaesthetic agents, including propofol. All of the previous studies used premedicated patients as well as a variety of agents in addition to propofol which could directly or indirectly affect the intraocular pressure. Most importantly the previous studies used high dose $(2 \cdot 0-2.5 \mathrm{mg} / \mathrm{kg})$ propofol which causes anaesthesia and has an increased risk of serious side effects. This study was undertaken to investigate the effect on intraocular pressure of a single low dose propofol bolus used for sedation in non-premedicated patients. Furthermore, the heart rate and blood pressure were monitored so that they could be correlated with changes in the intraocular pressure and because this information will be helpful in the clinical use of this drug.

\section{Materials and methods}

This study was conducted with institutional review board approval and informed consent was obtained from all participants. Twenty patients who were scheduled to undergo cataract surgery were enrolled. All patients 
with glaucoma, a history of allergic reaction to propofol, or other conditions preventing them from receiving propofol were excluded.

Patients were not given preanaesthetic sedatives. The contralateral non-study eye was dilated with cyclopentolate $1 \%$, phenylephrine (neosynephrine) $2.5 \%$, and flurbiprofen $0.03 \%$, each given as one drop 5 minutes apart for three doses beginning 1 hour before surgery. In the operating room, an intravenous catheter was placed in the patient's hand or arm and $50 \mathrm{mg}$ of intravenous lignocaine (xylocaine) was given to lessen the irritation from the administration of propofol. Patients received a mean bolus dose, $0.98 \mathrm{mg} / \mathrm{kg}$ (SEM 0.04 ), of propofol over 5 to 10 seconds. While the patients were sedated, a retrobulbar injection and facial nerve block were performed using $4 \mathrm{ml}$ of local anaesthetic for the former and $8 \mathrm{ml}$ of local anaesthetic for the latter. The local anaesthetic was drawn from a mixture of $10 \mathrm{ml}$ of $2 \%$ lignocaine, $10 \mathrm{ml}$ of $0.75 \%$ bupivacaine (marcaine), and 150 units of hyaluronidase. This was administered by the surgeon to the non-study eye approximately 1 to 2 minutes after propofol induction.

The intraocular pressures were measured in the eye that was not to undergo surgery. Before propofol induction, the study eye was anaesthetised with one drop of proxymetacaine $(0.5 \%)$ (proparacaine) and the intraocular pressure was measured. After propofol induction, the intraocular pressures were measured at 1 minute intervals for 5 to 7 minutes, depending upon the length of sedation. At the end of surgery, the study eye was anaesthetised again with one drop of proparacaine $(0.5 \%)$ and the intraocular pressure was measured. All measurements were made by the same investigator with the patient supine using a hand held applanation tonometer (Tonopen XL Bio$\mathrm{Rad})$. This instrument was calibrated at the beginning of each day as recommended by the manufacturer. The tonometer gives the mean intraocular pressure based upon four valid readings along with the reliability (\% standard

Table 1 Patient characteristics. Values expressed as mean (SEM) or as patient number, as appropriate

\begin{tabular}{lc}
\hline Age (years) & $68 \cdot 7(2 \cdot 2)$ \\
Weight (kg) & $81 \cdot 8(3 \cdot 2)$ \\
Sex (M:F) & $20: 0$ \\
Race (W:B) & $18: 2$ \\
ASA (I:II:III) & $2: 7: 11$ \\
Propofol dose (mg/kg) & $0.98(0 \cdot 04)$ \\
Surgery time (minutes) & $71 \cdot 5(16 \cdot 7)$ \\
\hline
\end{tabular}

*American Society of Anesthesiologists classifications.

Table 2 Mean intraocular pressure (SEM) ( $m m \mathrm{Hg}$ ) during intravenous sedation with propofol

\begin{tabular}{ll}
\hline & Propofol $(n=20)$ \\
\hline Preinduction & $16 \cdot 2(0 \cdot 7)$ \\
Propofol given (minutes) & \\
0 & \\
1 & $13 \cdot 1(0 \cdot 9)^{\star}$ \\
2 & $13 \cdot 4(0 \cdot 9)^{\star}$ \\
3 & $12 \cdot 8(0 \cdot 6)^{\star}$ \\
4 & $12 \cdot 3(0 \cdot 7)^{\star}$ \\
5 & $11 \cdot 8(0 \cdot 7)^{\star}$ \\
6 & $12 \cdot 1(0 \cdot 7)^{\star}$ \\
7 & $12 \cdot 2(0 \cdot 7)^{\star}$ \\
After surgery (71.5(16.7) minutes) & $16 \cdot 4(0 \cdot 6)^{\star}$ \\
\hline
\end{tabular}

${ }^{\star} \mathrm{p}<0.001 ; \uparrow_{\mathrm{n}}=16 ; \neq \mathrm{n}=15$. deviation) of the readings. Most of the Tonopen measurements were $<5 \%$ SD $(88 \%)$ and the rest were $<10 \% \mathrm{SD}$.

The heart rate was monitored continuously using a North American Drager-Markomed III unit and only the values which corresponded with intraocular pressure were recorded. The systolic and diastolic blood pressures were measured with a non-invasive blood pressure monitor (North American Drager-Markomed III unit) before and during induction.

During sedation, the patient was monitored for signs of airway obstruction by an anaesthetist and if significant airway obstruction occurred the airway was maintained by the chin lift technique. Each patient received 2 litres of oxygen by nasal cannula during sedation and surgery.

Computerised statistical analysis was performed using SAS statistical software by the Indiana University Department of Biostatistics. The intraocular pressure and pulse were analysed by the repeated measures analysis of variance test looking at the difference in time for intraocular pressure and pulse. This test allows for the differences in baselines of these patients to be taken into account. The lowest systolic and diastolic blood pressures of each patient were averaged and then analysed by the paired $t$ test so that individual variations in baselines could be accounted for. $p$ Values less than 0.01 were considered to be significant.

\section{Results}

Table 1 shows the characteristics of the 20 male patients aged 46 to 84 years and weighing between 50 and $120 \mathrm{~kg}$. The patients received a single bolus of $0.98 \mathrm{mg} / \mathrm{kg}$ (SEM 0.04 ) of propofol.

The mean intraocular pressures before propofol induction, at 1 minute intervals after induction, and after surgery are shown in Table 2. There was a significant $(p<0.001)$ change in the mean intraocular pressure for at least 7 minutes following induction with propofol. Table 2 shows a decrease in mean intraocular pressure of $17 \%$ to $27 \%$ from baseline after propofol induction. There is no significant difference $(p=0.71)$ between the preoperative mean intraocular pressure and the postoperative mean intraocular pressure (Table 2).

Figure 1 depicts the change of the mean intraocular pressure before, during, and after intravenous sedation with propofol. This

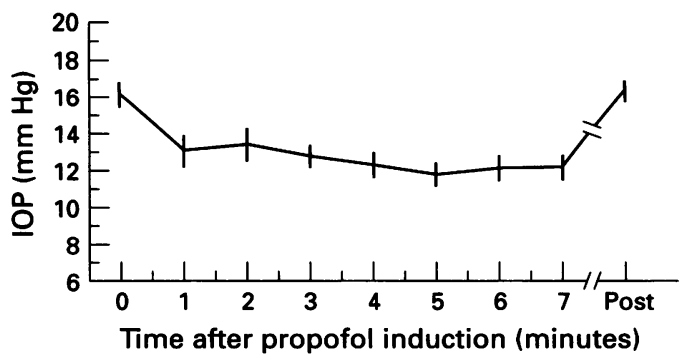

Figure 1 Mean intraocular pressure before and after intravenous sedation with propofol. 
Table 3 Mean pulse rate (SEM) (beats per minute) during intravenous sedation with propofol

\begin{tabular}{ll}
\hline & Propofol $(n=20)$ \\
\hline Preinduction & $72 \cdot 1(3 \cdot 3)$ \\
Propofol given (minutes) & \\
0 & \\
1 & $80 \cdot 9(3 \cdot 3)^{\star}$ \\
2 & $79 \cdot 6(3 \cdot 5)^{\star}$ \\
3 & $78 \cdot 2(3 \cdot 1)^{\star}$ \\
4 & $77 \cdot 0(3 \cdot 0)^{\star \star}$ \\
5 & $76 \cdot 4(2 \cdot 9)$ \\
6 & $74 \cdot 4(3 \cdot 5) \dagger$ \\
7 & $74 \cdot 2(3 \cdot 6) \ddagger$ \\
After surgery & $70 \cdot 4(2 \cdot 6)$ \\
\hline
\end{tabular}

${ }^{\star} \mathrm{p}<0.001,{ }^{\star \star} \mathrm{p}<0.01 ; \mathrm{fn}=16 ; \mathrm{fn}=15$.

illustrates that propofol decreases the intraocular pressure within the first minute after it is given. There is a slight increase in the mean intraocular pressure at 2 minutes compared with the 1 minute reading, but this is not statistically significant. The intraocular pressure continued downward to its lowest mean value of 11.8 at 5 minutes and remained significantly less than the preoperative intraocular pressure at 7 minutes when the measurements were terminated so that the patients could proceed with surgery.

Table 3 shows a statistically significant $(p<0.001$ to $<0.01$ ) increase of $7 \%$ to $12 \%$ in the mean pulse rate from the preinduction baseline during the first 4 minutes after propofol induction. There was no significant difference between the preinduction and the postinduction pulse rates at 5 minutes, 6 minutes, 7 minutes, and after surgery.

Table 4 shows a significant $(p<0.001)$ decrease of $12 \%$ in the mean systolic blood pressure and a significant $(\mathrm{p}<0.001)$ decrease of $7 \%$ in the mean diastolic blood pressure from the preinduction baseline level after propofol induction. The difference between the preinduction mean systolic and diastolic blood pressures was not significant $(p=0.14$ and $p=0.51$ respectively) when compared with the post-surgery mean systolic and diastolic blood pressures (Table 4).

Adequate sedation was achieved in all patients. The level of sedation for most patients was characterised by a sleeplike state with eyes closed and the patients were able to respond to verbal and tactile stimuli. The sedative effects of the low dose intravenous propofol bolus lasted between 2 and 5 minutes with the patient returning rapidly to a clear thinking state. The decrease in intraocular pressure continued after the sedative effects of propofol had ceased.

Table 4 Mean systolic and diastolic blood pressure (SEM) (mm Hg) during intravenous sedation with propofol

\begin{tabular}{lc}
\hline & Propofol $(n=20)$ \\
\hline Systolic blood pressure & \\
Preinduction & $151 \cdot 7(4 \cdot 8)$ \\
After propofol & $134 \cdot 0(4 \cdot 0)^{\star}$ \\
After surgery & $147 \cdot 1(3 \cdot 6)$ \\
Diastolic blood pressure & $80 \cdot 7(2 \cdot 4) \dagger$ \\
Preinduction & $74 \cdot 1(2 \cdot 3)^{\star}$ \\
After propofol & $81 \cdot 1(2 \cdot 1)$ \\
After surgery & \\
\hline${ }^{\star} \mathrm{p}<0 \cdot 001 ;+\mathrm{n}=19$. &
\end{tabular}

\section{Discussion}

This study finds that a single low dose $(0.98$ $\mathrm{mg} / \mathrm{kg}$ ) bolus of propofol used for intravenous sedation produces a significant decrease in intraocular pressure. The intraocular pressure decrease of $17 \%$ to $27 \%$ was less than the $31 \%$ to $53 \%$ decrease found in previous studies which used high dose $(2 \cdot 0-2.5 \mathrm{mg} / \mathrm{kg})$ propofol in combination with other anaesthetic agents. ${ }^{457}$ Several other studies found that there was a significant decrease in intraocular pressure from baseline before, and during intubation, when the combination of high dose $(2 \cdot 0-2 \cdot 5 \mathrm{mg} / \mathrm{kg})$ propofol, a neuromuscular blocker, a preanaesthetic benzodiazepine, and various other agents were administered to patients. ${ }^{4-68}$ Besides using propofol for induction before intubation, another study showed that high dose propofol $(2.2 \mathrm{mg} / \mathrm{kg})$ and $63 \%$ nitrous oxide reduced intraocular pressure by a maximum of $53 \%$ in elderly patients. ${ }^{7}$ Furthermore, this study found that propofol's effect of decreasing intraocular pressure lasted longer than its sedative effect. Also, the intraocular pressure was significantly decreased from the preoperative baseline at 7 minutes when the intraocular pressure readings were stopped so that the patient could proceed with surgery. It is likely that the intraocular pressure continued below the preoperative baseline for several more minutes and this would coincide with the beginning of surgery.

The mechanism by which propofol decreases intraocular pressure is postulated to be its ability to depress the ocular centres of the brain. ${ }^{79}$ Depression of these CNS ocular centres could cause a decrease in intraocular pressure by relaxing extraocular muscle tone, facilitating aqueous drainage, or both. ${ }^{79}$ Our study has demonstrated that there is an immediate decrease of the intraocular pressure after propofol induction. Therefore, it is unlikely that propofol is increasing aqueous humour drainage and even less likely that propofol is decreasing aqueous humour production. However, our study supports the hypothesis that propofol relaxes the extraocular muscle tone as a result of CNS depression. Previous studies suggest that propofol's side effect of causing hypotension is unlikely to be the major mechanism of the decrease in intraocular pressure. ${ }^{10-12}$ One laboratory study found that changes in systemic arterial pressure were poorly transmitted to the eye because of a dissociation between the iris artery pressure which had a high correlation with intraocular pressure and the ophthalmic artery pressure which had a high correlation with systemic arterial pressure. ${ }^{11}$ Another study reported that a large decrease in blood pressure up to $60 \%$ below the initial level was not accompanied by changes in intraocular pressure. ${ }^{10} \mathrm{~A}$ clinical study evaluating the effects of hypotensive anaesthesia found that in patients with an initial intraocular pressure over $11 \mathrm{~mm}$ $\mathrm{Hg}$ (all patients in this study) that there was no correlation between intraocular pressure and arterial pressure. ${ }^{12}$ Also, similar agents, such as thiopentone which at low doses have no 
significant effect upon blood pressure, still cause a decrease in intraocular pressure. ${ }^{7}$

Low dose intravenous propofol may be administered for the sedation of patients who are unable to cooperate for ocular examination such as young children and the mentally retarded. Propofol would be an excellent agent to select for this because of its previously mentioned properties. The effect of anaesthetic agents on intraocular pressure was important and must be considered when an examination under anaesthesia was performed and there was a concern about ocular hypertension. Based upon the results of this study, it can be surmised that a patient who receives a low dose bolus of propofol will have a moderate, approximately $20 \%$ to $25 \%$, decrease in intraocular pressure from the normal baseline level for at least 7 minutes after administration. Thus, if patients have a borderline high intraocular pressure or high normal intraocular pressure under anaesthesia then it is likely that they have ocular hypertension. This study was performed on elderly patients without ocular hypertension; however, it is possible that similar effects occur in children, patients with glaucoma, and patients with structural brain problems but this needs to be examined and confirmed by further study.

In addition to propofol's effect on intraocular pressure, it is important to consider its effect upon blood pressure and pulse rate. Low dose propofol was found to cause a mild decrease in systolic and diastolic blood pressure. Previous studies found that high dose propofol caused a moderate $(14 \%-19 \%)$ decrease in systolic blood pressure ${ }^{67}$ Propofol is thought to decrease blood pressure by directly depressing the peripheral artery and venous tone, decreasing myocardial contractility, resetting baroreceptor activity, and inhibiting sympathetic nervous system outflow. ${ }^{13}$ Our study showed that low dose propofol caused a short lived mild increase in heart rate after induction. This increase in heart rate is believed to be induced by the baroreceptor reflex in response to the decrease in blood pressure.

The disadvantages of using propofol include it causing a mild to moderate hypotension and a small increase in pulse rate; however, none of the study patients needed any treatment. In our study, propofol caused a mild, short lived respiratory depression caused by partial airway obstruction in six patients and this was easily managed by the anaesthetist with the application of a chin lift to maintain a patent airway. The side effects of propofol are dose dependent and they were easily managed at the lower dose. Another problem associated with propofol is that it causes a burning sensation when given intravenously. This can be minimised by anaesthetising the vein with a small amount of intravenous lignocaine before propofol induction. Another potential issue could be an eye that is too soft because of the decrease in intraocular pressure caused by propofol sedation. The two lowest intraocular pressure measurements in this study were 6 and $7 \mathrm{~mm}$ $\mathrm{Hg}$ in two different patients, and it is believed that these are not low enough to cause problems during the retrobulbar injections.

There were several possible sources of error in this study. One source of error is that all the patients had different baseline intraocular pressures, heart rates, and blood pressures. This problem has been minimised by using a repeated measures analysis of variance that allowed individual variations in baseline to be taken into account. Another source of error could be the use of a Tonopen instead of Goldmann tonometry. Studies have shown that Tonopens measure intraocular pressure accurately and that there is a good correspondence with Goldmann tonometry in the 11-20 $\mathrm{mm} \mathrm{Hg}$ interval and fairly good correspondence in the 4-10 $\mathrm{mm} \mathrm{Hg}$ and $21-30 \mathrm{~mm} \mathrm{Hg}$ intervals. ${ }^{14}$ Also, repeated measurements taken with a Tonopen could theoretically decrease the intraocular pressure because of repeated compression of the cornea with the instrument tip pushing the aqueous humour out of the eye and thus lowering the pressure. The probability of this occurring is thought to be minimal because measurements were taken by an experienced examiner using very light pressure to obtain the readings. Moreover, there was a levelling off of the intraocular pressure after propofol induction and one would expect that if this phenomenon were occurring, there would be a continued decrease in pressure with each measurement. Finally, the intravenous lignocaine used to numb the vein before propofol induction could theoretically cause changes in the intraocular pressure or alter propofol's effect upon intraocular pressure, but this was believed to be very unlikely because a low dose of lignocaine was used. Previous studies show lignocaine did not decrease intraocular pressure but did prevent the increase in intraocular pressure from noxious stimulus, such as intubation, by suppressing reflex responses. 1516

In conclusion, low dose propofol would be an excellent choice for sedation before ophthalmic surgery because of its beneficial sedative effects, minimal and easily controlled side effects, and the moderate decrease in intraocular pressure that occurs. Furthermore, it would be an appropriate choice for examination under anaesthesia and during this procedure is likely to cause a low to moderate decrease in intraocular pressure from baseline in most adult patients without glaucoma.

1 Bocian D, French S. Propofol (Diprivan) - a new intravenous anesthetic with applications for outpatient ambulatory surgery. Fournal of Foot Surgery 1992; 31: 603-6.

2 Deegan RI. Propofol: a review of the pharmacology and applications of an intravenous anesthetic agent. $A m \mathcal{F} M e d$ Sci 1992; 304: 45-8.

3 Ferrari LR, Donlon JV. A comparison of propofol, midazolam, and methohexital for sedation during retrobmidazolam, and methohexital for sedation during retrobMirakhur RK, Shepherd WFI, Darrah WC. Propofol or Mirakhur RK, Shepherd WFI, Darrah WC. Propofol or
thiopentone: effects on intraocular pressure associated thiopentone: effects on intraocular pressure associated (facilitated with suxamethonium). Br $\mathcal{F}$ Anaesth 1987; 59: 431-6.

5 Vanacker B, Dekegel D, Dionys J, Garcia R, Van Eeckhoutte L, Dralants G, Van de Walle J. Changes in intraocular pressure associated with the administration of propofol. Br F Anaesth 1987; 59: 1514-7.

6 Guedes Y, Rakotoseheno JC, Leveque M, Mimouni F, Egreteau JP. Changes in intra-ocular pressure in the elderly during anaesthesia with propofol. Anaesthesia 1988; 43: 58-60. 
7 Mirakhur RK, Shepherd WFI. Intraocular pressure changes with propofol (Diprivan): comparison with thiopentone. Postgrad Med f 1985; 61(Suppl 3): 41-4

8 Mirakhur RK, Shepherd WFI, Elliot P. Intraocular pressure sure changes during rapid sequence induction of anaesthesia: comparison of propofol and thiopentone in
combination with vercuronium. $B r \mathcal{F}$ Anaesth 1988; 60:

9 Murphy D. Anesthesia and intraocular pressure. Anesth Analg 1985; 64: 520-30

10 Schroeder M, Linssen GH. Intraocular pressure and anaesthesia. Anaesthesia 1972; 27: 165-70.

11 Macri FJ. Vascular and intraocular pressure. Arch Ophthalmol 1961; 65: 571-4

12 Tsamparlakis J, Casey TA, Howell W, Edridge A. Dependence of intraocular pressure on induced hypoten- sion and posture during surgical anesthesia. Trans Ophthalmol Soc UK 1980; 100: 521- 6

13 Searle N, Sahab P. Propofol in patients with cardiac disease. Can f Anaesth 1993; 40: 730-47.

14 Frenkel R, Young H, Shin D. Comparison of Tonopen to the Goldmann applanation tonometer. Arch Ophthalmol 1988; 106: 750-3

15 Mahajan R, Grover VK, Munjal VP, Hariwir S. Doubleblind comparison of lidocaine, tubocurarine, and diazepam pretreatment in modifying intraocular pressure increases. Can $\mathcal{F}$ Anaesth 1987; 34: 41-5.

16 Warner L, Bremer D, Davidson P, Rodgers G, Beach T. Effects of lidocaine, succinylcholine, and tracheal intubation on intraocular pressure in children anesthetized with halothane-nitrous oxide. Anesth Analg 1989; 69: 687-90. 